\title{
NanoZAF-A Pocket-Sized Utility for Microanalytical Experiment Design
}

\author{
Nicholas W. M. Ritchie \\ National Institute of Standards and Technology, Gaithersburg, MD 20899
}

When designing the optimal standards-based microanalysis measurement experiment, it is beneficial to understand how each of the different terms in the $C_{A}=Z \cdot A \cdot F \cdot k_{A}$ (where $k_{A}$ is the k-ratio of the A-th element) correction contributes to the calculation of sample composition. The three terms in the ZAF correction, the Z-term representing the correction due to backscatter and stopping power effects, the A-term representing the correction for X-ray absorption, and the F-term representing the correction for x-rays excited by fluorescence, depend on a semi-empirical model and many experimentally determined parameters. Some of these parameters are well characterized; others are less well characterized. To minimize the error in the resulting experiment as a result of the poorly characterized input parameters, it is best to select a standard with similar properties as the unknown. Alternatively when such a standard is not available, it is often necessary to select a binary material as a standard for a volatile or non-solid element. It is a poor idea to choose a binary material in which any one of the Z, A or F correction terms are significantly different from unity.

NanoZAF is a novel Palm ${ }^{\dagger}$ PDA (personal digital assistant) application to assist in this process. The user inputs the the instrument parameters (beam energy and take-off angle) and the estimated composition of the unknown or the known composition of the standard. The application computes the ZAF correction using the Pouchou and Pichoir PAP[1] model for each analytical line family (K, L and/or M) of each element in the sample relative to the correction for a pure elemental reference. The corrections are nominally unity when the behavior of the pure elemental reference is identical to the unknown. Of course, correction factors of unity are the ideal and typically the Aterm shows significant variation particularly for low energy lines or lines near the absorption edges of other sample elements. A-terms of $0.2-0.4$ are common for oxygen and depend strongly on the electron transport models and (relatively) poorly known mass absorption coefficients for oxygen. A similar statement can be made for other low energy lines - either K lines of light elements or L or M lines of heavier elements.

Knowledge of the correction factors can also assist the analyst in making intelligent choices selecting which lines to use to perform the composition calculation. Even if the intensity in a K-line is substantially less than the intensity in a L-line, it may be better to analyze using the $\mathrm{K}$ line if the correction factors for the K-line are closer to unity In addition, the analyst can consider how changing the beam energy will effect the ZAF correction. Lowering the beam energy can eliminate the fluorescence correction in some materials and reduce the absorption correction by reducing the average depth at which x-rays are generated. However, lowering the beam energy can also eliminate higher energy lines that typically have lower absolute mass absorption coefficients. The selection of optimal experimental parameters requires the kind of knowledge and understanding that NanoZAF can provide.

The PDA platform is ideal for this kind of utility. The portability of the platform encourages analysts to plan out their experiment before they sit down at the instrument. The user-defined sample database allows the analyst to record a list of available standards and deliberately select the optimal standard for each experiment. NanoZAF will be available for free through Palm Source at www.palmsource.com. NanoZAF requires a Palm compatible PDA with Palm OS version 3.0 or higher. 
Reference:

[1] J-L. Pouchou and F. Pichoir, Electron Probe Microanalysis, $1^{\text {st }}$ Ed., Plenum, New York, 1991

$\uparrow$ Certain commercial products are identified in this paper to foster understanding. Such identification does not imply recommendation or endorsement by the National Institute of Standards and Technology, nor does it imply that the products identified are necessarily the best available for the purpose.

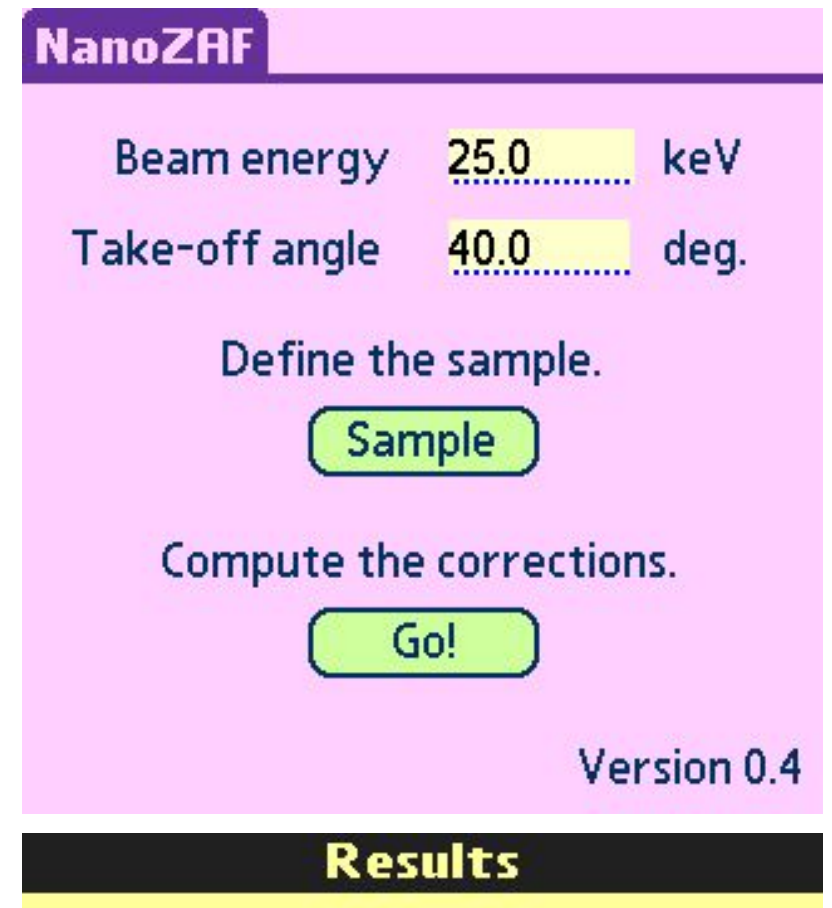

Sample K3189... at 25.0. keV

Take off angle 40.0..... degrees

Si-KA1: $1.0339 \times 0.5267 \times 1.0028$
Al-KA1: $1.0215 \times 0.4531 \times 1.0088$
Ca-KA1: $0.9355 \times 0.8940 \times 1.0175$
Mg-KA1: $1.0708 \times 0.3647 \times 1.00 \ldots$

\section{Si-KP1 at $1.740 \mathrm{keV}$}

$Z=1.034 \quad A=0.5267 \quad F=1.0028$ ZRF $=0.5460$

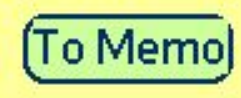

Done

\section{Define the sample}

Name $\nabla$ K3189

Weight \% $\quad$ Stoichiometry

$\begin{aligned} & 18.80 \% \text { Si by weight } \\ & 7.20 \% \mathrm{Al} \text { by weight } \\ & 14.40 \% \mathrm{Ca} \text { by wejpht } \\ & 5.20 \% \mathrm{Mg} \text { by weight }\end{aligned}$
Element .......... Quantity $0.00 . . . . \%$

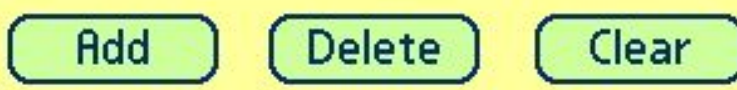

Density $5.5 \ldots . . . . . \mathrm{g} / \mathrm{cc} \quad$ Done

MAC

Radiation from element

Ca...

in

Sample

Ca KA1@ $3.691 \mathrm{keV}=307$

Ca LA1 @ $0.341 \mathrm{keV}=10403$

Figure: The primary input and results screens in NanoZAF. 